\title{
Hierarchical spring-block model for multiscale friction problems
}

\author{
Gianluca Costagliola, ${ }^{\dagger}$ Federico Bosia, ${ }^{\dagger}$ and Nicola M. Pugno ${ }^{*, \dagger, \uparrow}, \S$ \\ $\dagger$ Department of Physics and Nanostructured Interfaces and Surfaces inter-departmental Centre, University \\ of Torino, Via Pietro Giuria 1, 10125, Torino, Italy. \\ $\ddagger$ Laboratory of Bio-Inspired \& Graphene Nanomechanics, Department of Civil, Environmental and \\ Mechanical Engineering, University of Trento, Via Mesiano, 77, 38123 Trento, Italy \\ ISchool of Engineering and Materials Science, Queen Mary University of London, Mile End Road, London \\ E1 4 NS, UK \\ §Italian Space Agency, Via del Politecnico snc, 00133 Rome, Italy \\ E-mail: nicola.pugno@unitn.it
}

\begin{abstract}
A primary issue in bio-materials science is to design materials with ad-hoc properties, depending on the specific application. Among these properties, friction is recognized as a fundamental aspect characterizing materials for many practical purposes. Recently, new and unexpected frictional properties have been obtained by exploiting hierarchical multiscale structures, inspired by those observed in many biological systems. In order to understand the emergent frictional behaviour of these materials at the macroscale, it is fundamental to investigate their hierarchical structure, spanning across different length scales. In this paper, we introduce a statistical multiscale approach, based on a one-dimensional formulation of the spring-block model, in which friction is modeled at each hierarchical scale through the classical Amontons-Coulomb force with statistical dispersion on the friction coefficients of the microscopic components. By means of numerical simulations, we deduce the global statistical distributions
\end{abstract}


of the elementary structure at micrometric scale, and use them as input distributions for the simulations at the next scale levels. We thus study the influence of microscopic artificial patterning on macroscopic friction coefficients. We show that is it possible to tune the friction properties of a hierarchical surface and provide some insight on the mechanisms involved at different length scales.

Keywords: Friction, Multiscale modeling, Statistical properties, Hierarchy, Microscale structures

\section{Introduction}

Many biological systems commonly observed in Nature are organized accordin to a hierar-

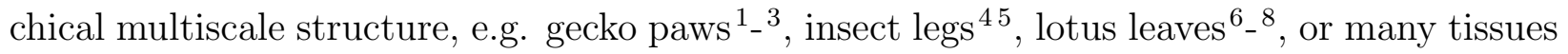

like bone, tendons, skin, etc ${ }^{9}[12$. These types of structures exhibit remarkable mechanical properties, which have attracted much interest in the quest of understand their underlying mechanisms and to design artificial materials with improved properties through complex multiscale structural organization of microscopic components ${ }^{13}[17$. This issue is inherently linked to research on bio-materials, in which it is essential to be able to tune the mechanical properties as desired. In particular, friction of bio-material surfaces is an important aspect that requires accurate characterization before a material can be practically employed for specific applications $18[22]$

In bio-inspired materials research new properties have been obtained by mimicking the structure observed in biological systems, suggesting that the key factor lies in the hierarchical architecture with interacting features at different size scales, whose combined effects lead to emergent synergistic properties ${ }^{23}$. This is true for friction of multi-structured surfaces, where intrinsic multiscale interactions can be combined with artificially introduced ones. Friction is an emergent complex phenomenon involving many microscopic degrees of freedom that combine in determining the macroscopic friction behaviour observed in experiments, and 
fully understanding friction in bio-inspired materials is still an open challenge.

The fundamental laws of friction were already established in XIX century in the framework of classical mechanics, with Amontons' law, stating that the friction force is proportional to the applied normal force and independent of the apparent contact surface, and Coulomb's law, stating that friction is independent of the sliding velocity ${ }^{24}$. However, since Galilei's time, friction was regarded as an effect to be eliminated in order to recover the predicted behaviour of the first principles, an unavoidable disturbance of the experiments involving any mechanical device. For this reason, the microscopic origin of the friction forces was scarcely investigated up to the second half of the XX century when, thanks to the advances in material physics, friction was recovered as active subject of research 25 .

Despite the apparent simplicity of the Amontons-Coulomb (AC) laws, it is difficult to construct a comprehensive theory for friction connecting the microscopic degrees of freedom to the macroscopic behaviour, since many different length scales and physical mechanisms are involved 26$]$, spanning from molecular adhesion forces to surface roughness contact forces $28[30$. Moreover, the basic description could be further complicated if thermal effects, wear and specific surface geometries were included, as occurs in bio-inspired materials. Thus, in order to accurately model and understand the basic mechanisms of these phenomena, many different approaches have been developed ${ }^{3132}$. On the one hand, the AC laws, which are valid only as a first approximation, can be improved by taking into account material elasticity and the velocity dependence in effective laws, as done in the work of Rice et al. ${ }^{[3]}$ or in $n^{34}\left[36\right.$. On the other hand, friction can be studied at atomic level ${ }^{[37}$, but linking these types of description with meso- or macroscopic models remains an open challenge in tribology. For this purpose numerical multiscale models have been proposed ${ }^{38[42}$. These methods consist in a combination of finite element methods (FEM) for large length scales and molecular dynamics methods for smaller ones, aiming to reconstruct the whole behaviour of the material during the sliding phase, from the microscopic contact slip to macroscopic elastic deformation. 
Another option consists in developing simplified models focused on the understanding of specific aspects of friction, e.g. the stick-slip behaviour ${ }^{\underline{43}}$ or the transition from static to kinetic friction ${ }^{44}$. These models can be solved numerically with reduced computational effort compared to $a b$ initio simulations and provide valuable insights on physical mechanisms, although they inevitably neglect other aspects in the aim to reduce the number of free parameters and usually involve only one length scale. The latter limitation can be overcome by implementing a hierarchical multiscale procedure, in which the statistical properties of the system obtained at a given scale level of simulation are used as input for the simulation of the next scale level.

These models have been already used, e.g. in fracture mechanics ${ }^{45146}$, where an initial statistical distribution is chosen for the rupture thresholds of the microscopic bonds to derive the global strength of the structure (e.g. a fiber bundle). The procedure can be repeated if many levels of hierarchy are present, simply by assuming that the local statistical properties of the next scale level are the resulting global properties of the previous one $e^{47}$. This kind of model can also be applied for friction, for which similar mechanisms have been observed ${ }^{48}$.

The main purpose of these statistical models is not necessarily to reconstruct the overall behaviour of the system, but mainly to highlight the mechanism acting in a multiscale system, in order to identify their features also in experimental tests. In particular, we consider materials which are artificially designed with patterned surface, e.g. grooves and pawls at different scales, aiming to understand how friction coefficients are affected by these surface features.

Recently we adopted a simple one-dimensional model based on the so-called spring-block model to understand the role of hierarchical patterning in friction, i.e. a sliding surface characterized by grooves of different length scales ${ }^{49}$. The spring-block model has already been used to investigate many different aspects of friction of elastic materials ${ }^{44150}[57$ and fits the requirements of simplicity and statistical treatment for a hierarchical friction model.

In this article, we propose a multiscale implementation of a one-dimensional version of the 
spring-block model: we consider a 1-D surface discretized in masses and springs, representing an elementary system in which the statistical dispersion is assigned to the microscopic friction coefficients. Regular or hierarchical patterning can be introduced at this level in order to simulate microscopic surface structures. Through iterated simulations we deduce the global frictional properties of the system, which are assigned as local statistical features of the next scale level. Thus, the influence of micro patterning at smaller scale levels on the properties at higher (macroscopic) levels is deduced. In this way it is also possible to study the friction properties of a composite material, in which the static and dynamic coefficients vary in the different segments of the surface.

The article is organized as follows: in section 2.1, we describe the model and in section 2.2 we introduce the multiscale approach; in section 3.1, we present the benchmark results for a single level, in 3.2 those for multiscale simulations with only one type of microscopic surface structuring; in the section 3.3 , we combine at the second level various microscopic surface patterns to show examples of the tuning of macroscopic friction properties; in section 4. we present the conclusions.

\section{1-D spring-block Model}

\subsection{Model formulation}

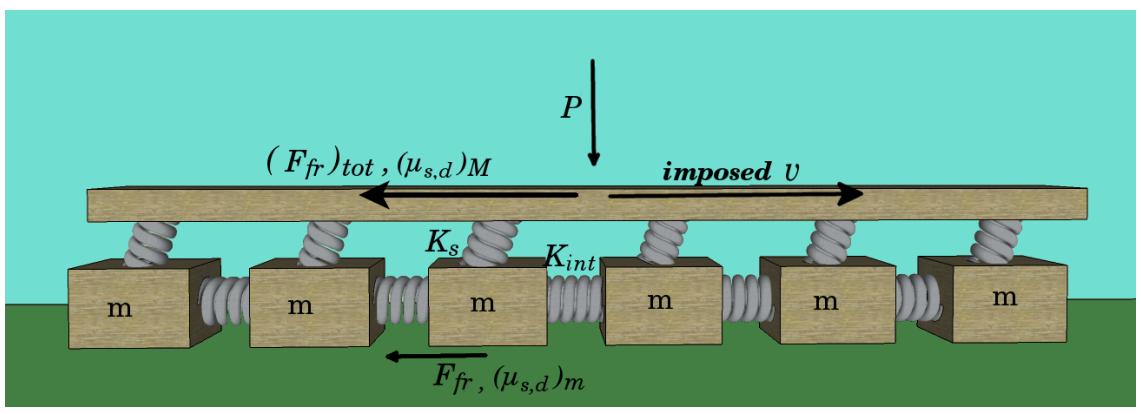

Figure 1: One-dimensional spring-block model with the notation used in the text.

We start from a one-dimensional formulation of the spring-block model ${ }^{49}$ (figure 1): 
we discretize a 1-D elastic surface into mass elements $m$ connected to their two nearest neighbours by springs of stiffness $K_{\text {int }}$. All the blocks are connected, through springs of stiffness $K_{s}$, to a slider, which is moving at constant velocity along the $x$ direction. Hence, the contact surface is discretized in $N$ blocks along the $x$-axis and the distance between blocks is $l$. This discretization is representative of the microscopic heterogeneity of the surface roughness.

In order to obtain the equivalence of the discretized system of masses and springs with an homogeneous elastic material, we assign the macroscopic quantities, i.e. the shear modulus $G=5 \mathrm{MPa}$, the Young's modulus $E=15 \mathrm{MPa}$, the mass density $\rho=1.2 \mathrm{~g} / \mathrm{cm}^{3}$ (typical values for an incompressible rubber-like material with Poisson ratio $\nu=0.5$ ). These are related to the stiffnesses $K_{\text {int }}=E l_{y} l_{z}(N-1) / L, K_{s}=G l_{y} l / l_{z}$, where $l_{y}$ and $l_{z}$ are the transversal dimensions and $L=N l$ is the total length, and to the mass of the blocks $m=\rho l_{y} l_{z} l$. The blocks are in contact with an infinitely rigid plane and are subjected to a constant and uniform pressure $P$. Hence, the normal force acting on each block is $F_{n}=l_{y} l P$. A viscous force with damping coefficient $\gamma$ is added in order to damp artificial block oscillations.

The interaction between the blocks and the plane is modeled by using a classical AC friction force $F_{f r}$ : when the block is at rest, the friction force exactly balances the horizontal force up to the static friction threshold, $F_{f r}=\mu_{s} F_{n}$. When the block starts to move, a constant force $F_{f r}=\mu_{d} F_{n}$ opposes the motion, where we have defined the static and dynamic friction coefficients as $\mu_{s}$ and $\mu_{d}$, respectively. In the following, we will drop the subscript $s$ and $d$ any time the considerations are referred to both the coefficients. In order to take into account the randomness of the surface asperities, the microscopic friction coefficients are chosen for each block from a Gaussian statistical distribution with mean value $(\mu)_{m}$ and standard deviation $\left(\sigma_{\mu}\right)_{m}$.

The total friction force $\left(F_{f r}\right)_{t o t}$ is obtained through the sum of all the friction forces acting on the blocks. Thus, the macroscopic friction coefficients are obtained by dividing it 
for the total normal force $\left(F_{n}\right)_{t o t}$, and they will be denoted as $(\mu)_{M}$.

The presence of patterning along the surface can be simulated by locally setting to zero the friction coefficients of some blocks corresponding to "grooves". Complex geometries can also be simulated with this approach, enabling the determination of the corresponding friction coefficients ${ }^{49}$.

The equation of motion along the sliding direction for the block $i$ can be written as: $m \ddot{x}_{i}=F_{\text {int }}+F_{s}-m \gamma \dot{x}_{i}+F_{f r}$, where $F_{f r}$ is the friction force defined previously, $F_{\text {int }}=$ $K_{\text {int }}\left(x_{i+1}+x_{i-1}-2 x_{i}\right)$ is the force between blocks and $F_{s}=K_{s}\left(v t+l_{i}-x_{i}\right)$ is the shear force due to the slider ( $l_{i}$ is the initial rest position of the block). Thus, we have a system of equations of motion for the blocks, that can be solved numerically with a fourth-order Runge-Kutta algorithm. The friction coefficients of the blocks are randomly chosen at each run, so that averaging procedures after various repetitions of the simulation are needed to obtain the final result of an observable. We have checked that with an elementary time step of $h=10^{-8} \mathrm{~s}$ the systematic error due to integration is negligible with respect to the corresponding statistical uncertainty.

For the other parameters, we fixed the following values: pressure $P=1 \mathrm{MPa}$, transversal lengths $l_{y}=1 \mathrm{~cm}, l_{z}=0.1 \mathrm{~cm}, \gamma=10 \mathrm{~ms}^{-1}$. The slider velocity is set to $v=0.1 \mathrm{~cm} / \mathrm{s}$, which is much smaller than the characteristic velocity $v_{s} \equiv l \sqrt{K_{\text {int }} / m}$ of the spring-mass system $\left(v / v_{s} \simeq 8 \cdot 10^{-6}\right)$. Although the dynamic friction coefficient in general depends on the sliding velocity, for velocities of this order of magnitude or smaller, which are typical of most experiments in the literature ${ }^{\sqrt{5015859}}$, results are not velocity-dependent, confirming those in ${ }^{60}$ obtained with a more detailed model. The microscopic friction coefficients are assumed at the first level to be $\left(\mu_{s}\right)_{m}=1.0(1)$ and $\left(\mu_{d}\right)_{m}=0.50(5)$, where we denote in round brackets the standard deviations of their Gaussian distributions. Their value is conventional, but actually only their ratio influences the results, e.g. multiplying both for the same numerical factors results in a global friction coefficient multiplied by the same factor.

Finally, the microscopic length $l$ can be associated in a real case to the average distance 
between the contact points due to the surface roughness or due to the smallest size of the textures designed on the material surface. We use $l=0.01 \mathrm{~cm}$, which falls in the range between one micrometer and one millimeter usually considered in typical applications 61 63 and in bio-inspired materials $\left[64\left[66\right.\right.$. In $\frac{49}{6}$ a full discussion of the role of this parameter in the spring-block model is presented. Essentially, since the stiffnesses depend on $l$, the are two regimes: $K_{\text {int }}>K_{s}$ in which the static to dynamic transition is dominated by avalanche ruptures and $K_{\text {int }}<K_{s}$ in which it is similar to a stochastic failure process. Having fixed the other parameter values, the model is always in the regime $K_{\text {int }}>K_{s}$ and the results are not affected significantly while $l$ varies in this range. These will be considered as the default parameters, while the number $N$ of blocks will be specified for each considered case.

\subsection{Multiscale approach}

The multiscale approach illustrated in figure 2 requires that the macroscopic quantities related to an ensemble at the lower scale level become the microscopic quantities of an elementary unit at the next scale level. For example, by repeating the simulations on a chain of $N^{(1)}$ blocks of size $l^{(1)}$ representing the first level, we obtain a distribution of the global friction coefficients for this ensemble. Hence, at the second level, we impose that the elementary block size is $l^{(2)}=N^{(1)} \cdot l^{(1)}$ and its microscopic friction coefficients are randomly extracted from the global distribution found at the previous level. Similarly, the mass is scaled as $m^{(2)}=m^{(1)} N^{(1)}$. The stiffnesses can be deduced from the formulas in section 2.1, $K_{\text {int }}^{(2)}=K_{\text {int }}^{(1)} / N^{(1)}$ and $K_{s}^{(2)}=K_{s}^{(1)} N^{(1)}$ (we assume that the Young's modulus and the shear modulus are constant). This behaviour is peculiar to the one-dimensional model and imply that, when increasing the scale level, $K_{\text {int }}$ becomes smaller, i.e. the internal forces become less relevant. This process in principle could be iterated many times for every scale needed to describe the material.

This multiscale approach has various advantages: firstly, it is possible to simulate different scales of roughness on the surface, or to introduce micro-structures at the smaller scale in 
order to study how they influence the global behaviour. For example, by setting to zero the friction coefficients of certain blocks, we can simulate a microscopic patterning of grooves and pawls at the first level and determine the global friction coefficients of this ensemble, to be assumed as the elementary ones of the next scale level (as shown in figure 2). Secondly, to obtain results for the higher levels, it is not necessary to repeat the simulations at the previous level: once its distribution of the global friction coefficient has been calculated with a good approximation, the microscopic coefficients of the next level are extracted directly from this distribution. This avoids the need to take into account all the elements at smaller scales for every single simulation, which would imply a large computational time. In the following, we will present the results for elementary systems, showing how friction coefficient distributions change as a function of patterning at microscopic level. 


\section{1-st level: Elementary Units}

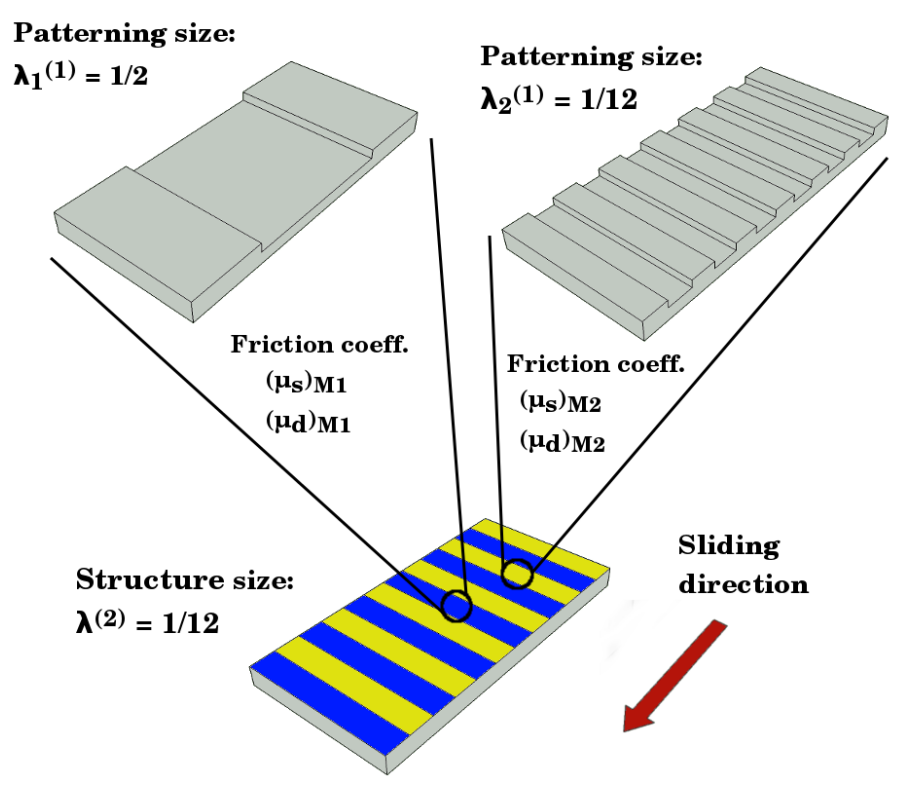

2nd-level: Composite Surface

Figure 2: Example of the modelization of a two-level multiscale structure: at the first level there is patterning with two different periods or groove sizes (we denote with $\lambda^{(1)}$ the ratio between the groove size and the total size of the elementary unit). These are repeated in succession as shown in the figure at the second level, where $\lambda^{(2)}$ is the size of the structures. The friction coefficient distributions of the elementary units, deduced by means of iterated simulations of the 1-D spring-block model in case of patterning, are used as input for the second level. The procedure can be iterated for each scale level required to describe the material. 


\section{Results}

\subsection{Single-level simulations}

In this section, we present the results for a single hierarchical level, in which surfaces are patterned in grooves and pawls (see upper part of figure 2). We start from a regular periodic patterning with grooves of size $l_{g}$ placed at the same distance each other, so that half of the surface remains in contact. Given the total length of the units $L$, we denote the configuration with the ratio $\lambda \equiv l_{g} / L$. We report results of simulations for elementary units with the default system parameters of section 2.1, length $l=0.01 \mathrm{~cm}$, number of blocks $N=24$, hence the total length of the units is $L=0.24 \mathrm{~cm}$. The comparison of the total friction force divided by the normal force, as a function of time, between smooth and patterned surfaces is shown in figure 3: the static friction coefficient is calculated from the first peak of the friction force, whilst the dynamic friction coefficient is calculated from the average over the sliding phase. The statistical distributions are obtained by performing about $10^{3}$ repeated simulations.

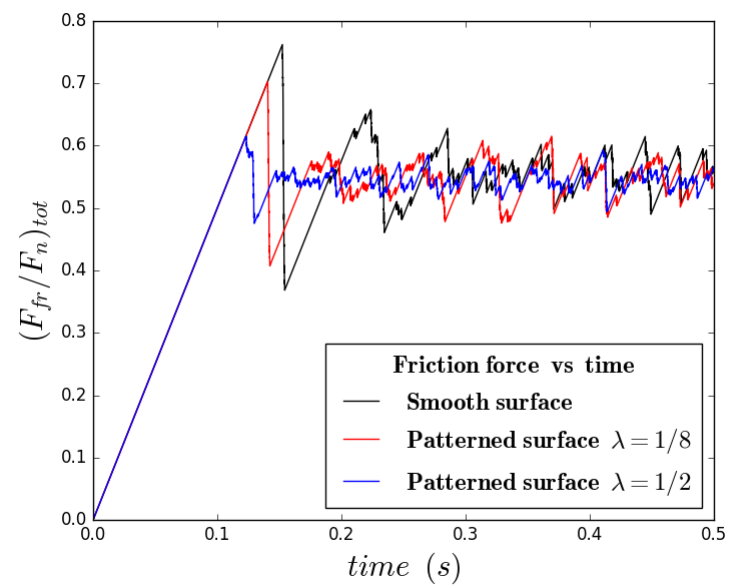

Figure 3: Comparison of the total friction force as a function of time between different single-level units.

Results are consistent with those from the literature 495767 , i.e. the static friction coefficient is reduced for larger grooves, while the dynamic coefficient is virtually unchanged (figure $4 \mathrm{a}$ ). This can also be observed also in the statistical distributions (figure $4 \mathrm{~b}$ ) of the 
macroscopic coefficients obtained with iterated simulations for the elementary units: the dynamic coefficient distribution is practically identical, while for static coefficients, in the case of large patterning, is shifted to a smaller value. The variance is reduced and the symmetry around the mean value is lost, and this is much more evident when the static friction distribution is close to that of the dynamic one. We have also tested a single-level random configuration, i.e. half the blocks randomly chosen have friction coefficients set to zero to simulate a random pattern of asperities. The results are similar to the case of larger grooves with static friction severely reduced.

Results show that the statistical distribution of friction coefficients can be modified by acting on the geometry of the surface, without modifying the material properties. In $\frac{\sqrt[49]{9}}{w e}$ showed that by introducing hierarchical patterning, i.e. grooves of different size scales, is it possible to manipulate the static friction coefficients of a surface, obtaining, for example, a smaller static friction coefficients with a small contact area. Hence, it is possible to obtain elementary units with tailor-made statistical friction properties. In the next section, we will show how they can be combined to simulate a composite material with different types of structures on the surface. The friction properties of this system can be deduced by means of the multiscale approach described in section 2.2 .

\subsection{Multi-level simulations}

Let us first consider only one type of elementary unit repeated along the whole surface at the second scale level. From this structure it is possible to deduce the global statistical distribution of the friction coefficients, which can in turn be used as input for the third level, and so on. The surface displays patterning only at the lowest scale level. Using the described multiscale procedure it is possible to derive the scaling of friction properties, by simulating their variation for increasing total system size with respect to the smallest scale at which heterogeneity and patterning appear. As example, we study the behaviour of the five microscopic structures of figure 43 with the default set of parameters, $N^{(1)}=24$ and 

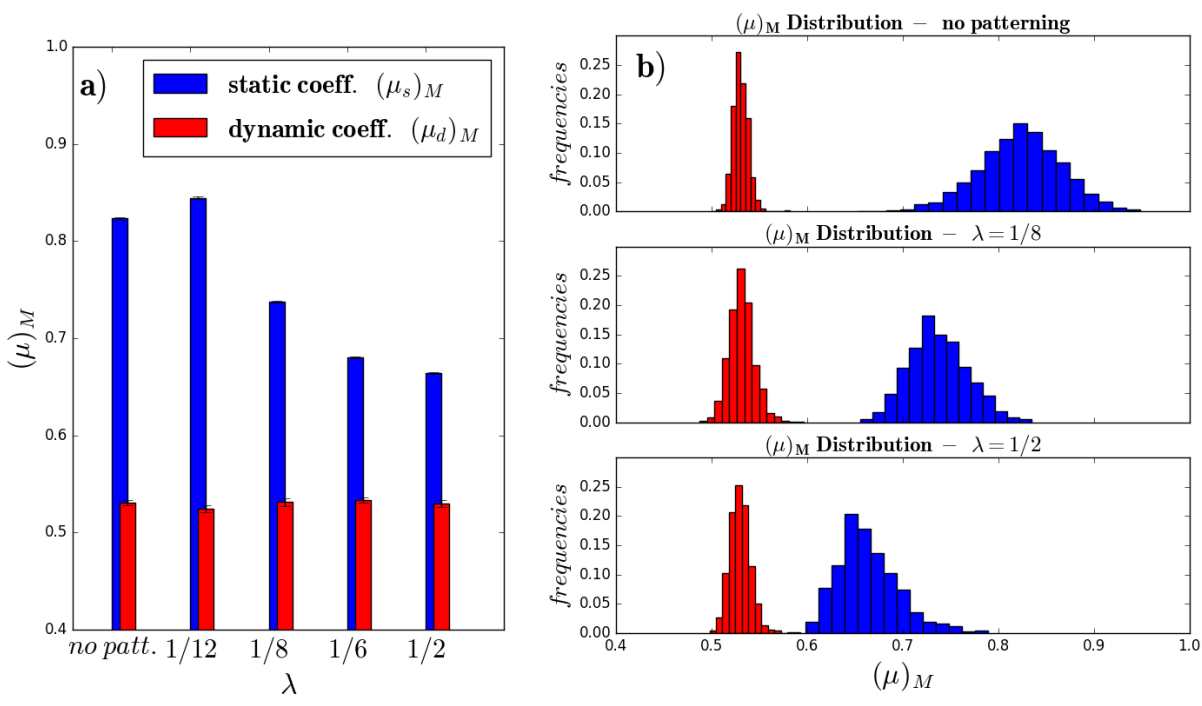

Figure 4: a) Comparison of the friction coefficients by varying the groove size, i.e. the ratio $\lambda$. The static friction coefficient decreases for larger grooves while the dynamic one remains constant. These results are obtained on systems with the default set of parameters and a total size $L=0.24 \mathrm{~cm}$. b) Statistical distributions of the global friction coefficients $\mu_{M}$ for elementary units for the case without patterning and the cases $\lambda=1 / 8$ and $\lambda=1 / 2$.

$l^{(1)}=0.01 \mathrm{~cm}$. The results for the next levels are obtained with the parameters scaled as explained in section 2.2, and number of blocks $N^{(2)}=20, N^{(3)}=12$.

Results are shown in figure 5: the static friction is reduced by increasing the number of hierarchical levels, i.e. the total size of the system. This is consistent with results from $\underline{49}$ and is a statistical effect, similarly to the reduction of strength with increasing specimen size observed in fracture mechanics ${ }^{45}$. This is also typical of static friction, which exhibits similar properties to fracture mechanics 48 .

On the other hand, the dynamic friction coefficient slightly increases with system size but, while for single-level ensembles we do not observe differences by varying the groove size, from the second scale level dynamic friction is mostly increased for a non patterned surface and small $\lambda$ (see figure 5e). This is because dynamic friction is influenced by the statistical distribution of the static one. During the system motion a fraction of the blocks is at rest and subjected to static friction, so that we expect a higher dynamic friction in presence of a greater value of the static one. This is more evident from the second level, where the 
static friction distributions assumed at the microscopic level have different shapes due to the patterning of the surface (figure $4 \mathrm{~b}$ ). From this we can deduce that the effect of larger grooves at microscopic level is to reduce dynamic friction at macroscopic scales.

Finally, the standard deviations of the distributions decrease with increasing levels (figures 5b,d): this effect is due to statistics, since fluctuations become less influential with an increase in size of the sample. However, this has also the effect to decrease the relative reduction of the static friction for higher levels, as observed in figure 5 a, which is due to the width of the statistical distribution.
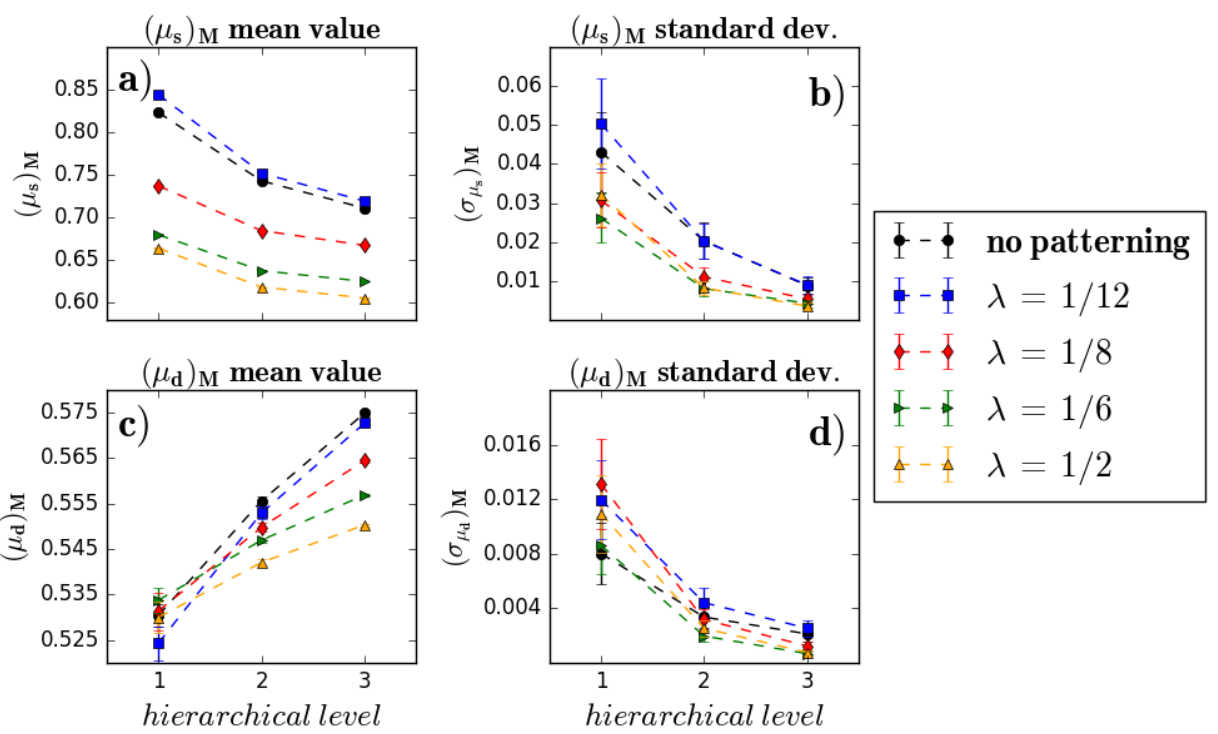

Figure 5: Scaling of mean values (left) and standard deviations (right) of the macroscopic friction coefficient distributions as a function of the hierarchical level in the multiscale procedure, for different types of elementary units, i.e. patterned surfaces. 


\subsection{Multiscale structured surfaces}

We now construct the second level configurations by combining different types of elementary units. In other words, the elementary components of the second level have friction coefficients assigned from the probability distributions of the elementary units studied in section 3.1 . arranged in regions of variable length, as depicted in figure 2. In this example, units with small grooves are combined with larger ones, but other combinations can be designed, for example smooth regions (without patterning) and patterned regions with a fixed $\lambda^{(1)}$. Mean and variance of the distributions are taken from the results of section 3.1 ,

For simplicity we will consider here only the combinations similar to those shown in figure 2. i.e. zones of length $l_{g}^{(2)}$ with different elementary units separated by the same distance $l_{g}^{(2)}$. Given the total length at the second level $L^{(2)}=N^{(2)} l^{(2)}$, we denote the second level configurations with the ratio $\lambda^{(2)}=l_{g}^{2} / L^{(2)}$.

Results are reported in figure 6 and show how it is possible to tune the global properties only by changing the microscopic arrangement of the structures. When regions with different friction coefficients are created (figure 6 right), the resulting static friction coefficient is closer to the smaller value of the two, instead of being close the average, as one could expect. This effect is due to the different average static thresholds of the two regions: when the weaker ones are exceeded, the configuration of the system is analogous to that with grooves and pawls at elementary level, so that the static friction is reduced. A similar trend is observed by increasing $\lambda^{(2)}$.

Instead, if we combine elementary units with a smooth surface (i.e. without patterning) and with small grooves (as in figure 6 left, in which $\lambda^{1}=1 / 12$ ), we observe at the second hierarchical level an increase of the static friction coefficient with respect the cases without combination discussed in section 3.2. In this case there is a non-trivial interaction of the regions with similar global static friction coefficient but with different statistical distributions.

The dynamic friction coefficients do not change significantly with $\lambda^{(2)}$, but they slightly increase with the same behaviour observed in section 3.2 . 

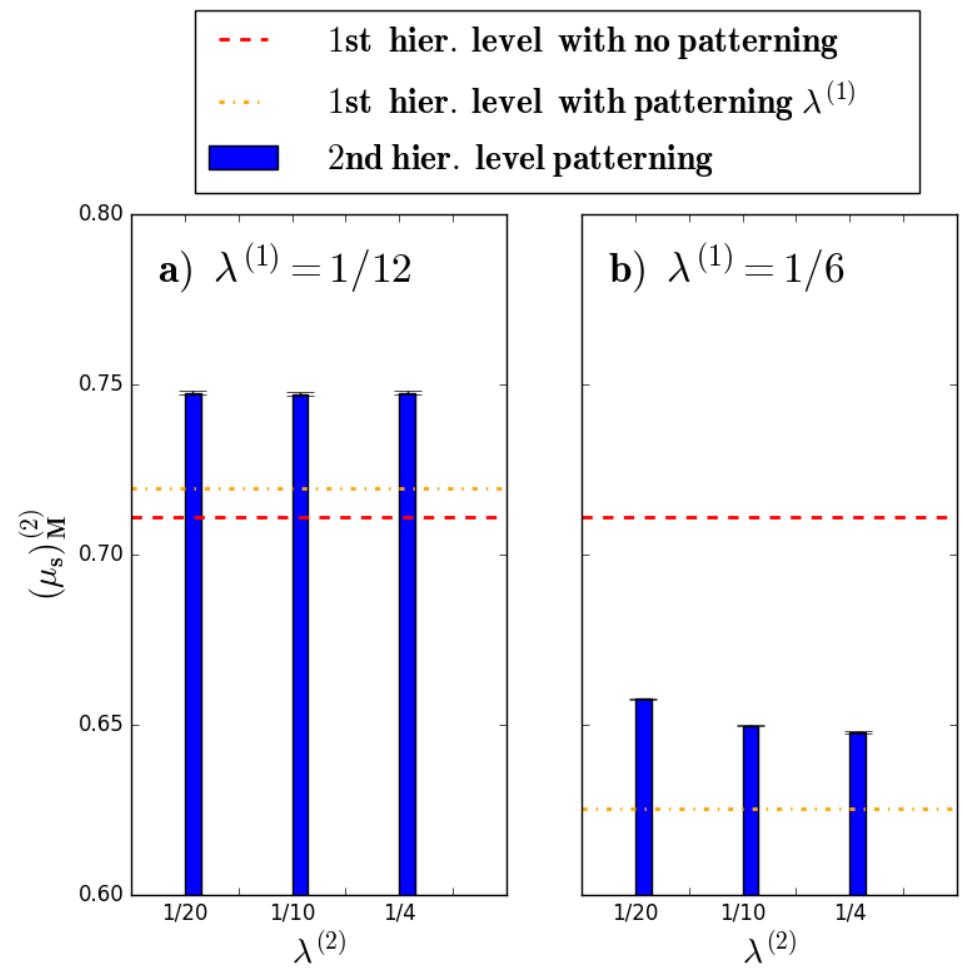

Figure 6: Comparison of the static friction coefficients for second-level hierarchical surfaces, structured as in figure 2, where there are alterning regions made of non patterned (smooth) elementary units and of grooves of size ratio $\lambda^{(1)}$. Results obtained in section 3.1 are included with dashed lines for comparison. These are relative to the second hierarchical level with the repetition of the same elementary units of the type specified in the legend. 


\section{Conclusions}

In this paper, we hierarchically extended the classical spring-block model to study the scaling of friction properties in the presence of surface structures at various size scale. This has been achieved using a one-dimensional formulation of the model, in which the surface roughness at a micrometric scale is simulated by randomly extracting the friction coefficients of each block from a statistical distribution. Surface patterning at various size scales has been simulated by removing friction from selected blocks. The resulting distributions of global friction coefficients for these units have been used as input for the scaled-up model at the next level. This kind of approach has already been used in fracture mechanics models, but we have also shown it to be appropriate for friction, where the transition from static to dynamic phase has many similarities with fracture nucleation phenomena.

We have investigated multiscale frictional behaviour in presence of patterning, recovering the previously known result that the static friction coefficient is reduced by grooves of increasing size. Moreover, by studying the behaviour induced by these elementary units at larger scale, there is a progressive reduction of static friction, as expected due to the increasing number of microscopic components. The growth of the length scale does not modify the effects of the grooves observed at the smallest scale. However, dynamic friction, which is unaffected by grooves at microscopic level, generally increases at higher scales, mostly for elementary units with smooth surfaces and smaller grooves. The resulting statistical distributions display a reduction of the variance with increasing length scale.

We have also considered a combination of two types of elementary units, to simulate for example a non patterned area combined with area with large grooves, alternating them at the second level. When the friction coefficients of the two units are different, the resulting static one is smaller than their average, showing a non trivial interaction. From this we can argue that, to considerably reduce static friction, it is sufficient to design microscopic structures, such as grooves, only on part of the surface. Dynamic friction is not affected by these kinds of combinations. 
These results show that is it possible to manipulate the friction properties at macroscopic level by means of micro structures on the surface. In general, we have shown that a multiscale statistical approach allows to highlight the fundamental mechanisms occurring across different length scales in the friction of structured hierarchical surfaces, such as those found in biological materials. Results can be useful for the design and development of novel biomaterials with tailor-made frictional properties.

\section{Acknowledgments}

N.M.P. is supported by the European Research Council PoC 2015 "Silkene" No. 693670, by

the European Commission H2020 under the Graphene Flagship Core 1 No. 696656 (WP14 "Polymer Nanocomposites") and FET Proactive "Neurofibres" grant No. 732344. G.C. and F.B. are supported by H2020 FET Proactive "Neurofibres" grant No. 732344.

\section{References}

(1) K. Autumn, Y. Liang, T. Hsieh, W. Zesch, W.-P. Chan, T. Kenny, R. Fearing, R.J. Full, Adhesive force of a single gecko foot-hair, Nature 405 (2000) 681

(2) B. Bhushan, Adhesion of multi-level hierarchical attachment systems in gecko feet, J. Adhesion Sci. Technol. 21 (2007) 1213

(3) M. Varenberg, N. M. Pugno, S.N. Gorb, Spatulate structures in biological fibrillar adhesion, Soft Matter 6 (2010) 3269

(4) T. Eisner, D. J. Aneshansley, Defense by foot adhesion in a beetle (Hemisphaerota cyanea), Proc. Natl Acad. Sci. USA 97 (2000) 6568

(5) N.M. Pugno, Spiderman gloves, Nano Today 3 (2008) 35 
(6) W. Barthlott, C. Neinhuis, Purity of the sacred lotus, or escape from contamination in biological surfaces Planta, 202 (1997) 1

(7) D. Quere, Fakir droplets, Nat. Mater. 1 (2002) 145

(8) S.S. Latthe, C. Terashima, K. Nakata, A. Fujishima, Superhydrophobic Surfaces Developed by Mimicking Hierarchical Surface Morphology of Lotus Leaf, Molecules 19 (2014) 4256

(9) H.J. Gao, Application of Fracture Mechanics Concepts to Hierarchical Biomechanics of Bone and Bone-like Materials, Int. J. Fract. 138 (2006) 101

(10) P. Fratzl, R. Weinkamer, Natures hierarchical materials Prog. Mater. Sci. 52 (2007) 1263

(11) J.D. Currey, Hierarchies in Biomineral Structures, Science 309 (2015) 253

(12) F. Fang, S.P Lake, Modelling approaches for evaluating multiscale tendon mechanics, Interface Focus 6 (2016) 1, 20150044

(13) R. Lakes, Materials with structural hierarchy, Nature 361 (1993) 511

(14) B. Ji, H.J. Gao, Mechanical properties of nanostructure of biological materials, J. Mechan. Phys. Solids 52 (2004) 9

(15) H.J. Gao, B. Ji, I.L. Jäger, E. Arzt, P. Fratzl, Materials become insensitive to flaws at nanoscale: Lessons from nature PNAS 103 (2003) 5597

(16) S.W. Cranford, A. Tarakanova, N.M. Pugno, M.J. Buehler, Nonlinear material behaviour of spider silk yields robust webs, Nature 482 (2012) 72

(17) J. Zang, S. Ryu, N.M. Pugno, Q. Wang, Q. Tu, M.J. Buehler, X. Zhao, Multifunctionality and control of the crumpling and unfolding of large-area graphene, Nature Materials $12(2013) 321$ 
(18) M. La Berge, J.B. Medley, J.M. Rogers-Foy, Friction and Wear, in Handbook of biomaterials evaluation edited by A.F. von Recum (1998)

(19) D.R. Millert, S. Das, K.Y. Huang, S. Han, J. N. Israelachvili, J.H. Waite, Mussel Coating Protein-Derived Complex Coacervates Mitigate Frictional Surface Damage, ACS Biomater. Sci. Eng. 1 (2015) 11, 1128

(20) S.A. Catledge, M.D. Fries, Y.K. Vohra, W.R. Lacefield, J.E. Lemons, S. Woodard, R. Venugopalan, Nanostructured ceramics for biomedical implants, J. Nanosci. Nanotechnol. 2 (2002) 293

(21) M. Berni, N. Lopomo N, G. Marchiori G, A. Gambardella A, M. Boi M, M. Bianchi, A. Visani, P. Pavan, A. Russo, M. Marcacci, Tribological characterization of zirconia coatings deposited on Ti6Al/4 Components for orthopedic applications, Mater. Sci. Eng. C Mater. Biol. Appl. 62 (2016) 643

(22) C.L. Kim, D.E. Kim, Self-healing Characteristics of Collagen Coatings with Respect to Surface Abrasion, Sci. Rep. 6 (2016) 20563

(23) S. Signetti, F. Bosia, N. M. Pugno, Computational modeling of the mechanics of hierarchical materials, MRS Bulletin, 41 (2016) 694

(24) B.N.J. Persson, Sliding Friction - Physical principles and application, in Nanoscience and Technology, Springer-Verlag Berlin Heidelberg (2000)

(25) M. Urbakh, E. Meyer, Nanotribology: The renaissance of friction, Nature Materials 9 (2010) 8

(26) M. Nosonovsky, B. Bhushan, Multiscale friction mechanisms and hierarchical surfaces in nano- and bio-tribology, Mater. Sci. Eng. R 58 (2007) 162

(27) M. Nosonovsky, B. Bhushan, Multiscale Dissipative Mechanisms and Hierarchical Surfaces, Springer-Verlag Berlin Heidelberg (2008) 
(28) V.A. Yastrebov, J. Durand, H. Proudhon, G. Cailletaud, Rough surface contact analysis by means of the Finite Element Method and of a new reduced model, Comptes Rendus Mecanique, 339 (2011) 473

(29) V.A. Yastrebov, G. Anciaux, J.F. Molinari, Contact between representative rough surfaces, Phys. Rev. E 86 (2012) 035601

(30) V.A. Yastrebov, G. Anciaux, J.F. Molinari, From infinitesimal to full contact between rough surfaces: evolution of the contact area, International Journal of Solids and Structures, $52(2015) 83$

(31) E. Gnecco, E. Meyer editors, Fundamentals of Friction and Wear on the Nanoscale second edition, Springer (2014)

(32) A. Vanossi, N. Manini, M. Urbakh, S. Zapperi, E. Tosatti, Colloquium: Modeling friction: From nanoscale to mesoscale, Rev. Mod. Phys. 85 (2013) 529

(33) J.R. Rice, N. Lapusta, K. Ranjith, Rate and state dependent friction and the stability of sliding between elastically deformable solids, Journal of the Mechanics and Physics of Solids 49 (2001) 1865

(34) J.R. Rice, A.L. Ruina, Stability of steady frictional slipping, J. Appl. Mech.50 (1983) 343

(35) J.H. Dieterich, B.D. Kilgore, Direct observation of frictional contacts: new insights for state dependent properties, Pure Appl.Geophys. 143 (1994) 283

(36) C.H. Scholz, Earthquakes and friction laws Nature 391 (1998) 37

(37) B. Bhushan, J. N. Israelachvili, U. Landman, Nanotribology: friction, wear and lubrication at the atomic scale Nature 374 (1994) 607

(38) B.Q. Luan, S. Hyun, J.F. Molinari, N. Bernstein, M.O. Robbins Multiscale modeling of two-dimensional contacts, Phys. Rev. E 74 (2006) 046710 
(39) G. Anciaux, J.F. Molinari, Contact mechanics at the nanoscale, a 3D multiscale approach, Int. J. Numer. Meth. Engng. 79 (2009) 9

(40) E. Weinan, W.Q. Ren, E. Vanden-Eijnden, A general strategy for designing seamless multiscale methods, J. Comput. Phys. 228 (2009) 5437

(41) Y. Mo, K.T. Turner, I. Szlufarska, Friction Laws at the Nanoscale, Nature 457 (2009) 1116

(42) Y. Liu, I. Szlufarska, Chemical Origins of Frictional Aging, Phys. Rev. Lett. 109 (2012) 186102

(43) M.G. Rozman, M. Urbakh, J. Klafter, Controlling chaotic frictional forces, Phys. Rev. E 57 (1998), 7340

(44) O.M. Braun, I. Barel, M. Urbakh, Dynamics of Transition from Static to Kinetic Friction, Phys. Rev. Lett. 103 (2009) 194301

(45) S. Pradhan, A. Hansen, B. K. Chakrabarti, Failure processes in elastic fiber bundles, Rev. Mod. Phys. 82 (2010) 499

(46) S. Zapperi, A. Vespignani, H. E. Stanley, Plasticity and avalanche behaviour in microfracturing phenomena, Nature 388 (1997) 658

(47) N.M. Pugno, F. Bosia, T. Abdalrahman, Hierarchical fiber bundle model to investigate the complex architectures of biological materials, Phys. Rev. E 85 (2012) 011903

(48) E. Bayart, I. Svetlizky, J. Fineberg, Fracture mechanics determine the lengths of interface ruptures that mediate frictional motion, Nature Physics 12 (2016) 166

(49) G. Costagliola, F. Bosia, N.M. Pugno, Static and dynamic friction of hierarchical surfaces, Phys. Rev. E 94 (2016) 063003 
(50) S. Maegawa, A. Suzuki, K. Nakano, Precursors of Global Slip in a Longitudinal Line Contact Under Non-Uniform Normal Loading, Tribol. Lett. 38 (2010) 3

(51) J. Scheibert, D.K. Dysthe, Role of friction-induced torque in stick-slip motion, EPL 92 (2010) 5

(52) J. Trømborg, J. Scheibert, D.S. Amundsen, K. Thøgersen, A. Malthe-Sørenssen, Transition from Static to Kinetic Friction: Insights from a 2D Model, Phys. Rev. Lett. 107 (2011) 074301

(53) E. Bouchbinder, E.A. Brener, I. Barel, M. Urbakh, Slow Cracklike Dynamics at the Onset of Frictional Sliding, Phys. Rev. Lett. 107 (2011) 235501

(54) R. Capozza, S.M. Rubinstein, I. Barel, M. Urbakh, J. Fineberg, Stabilizing stick-slip friction, Phys. Rev. Lett. 107 (2011) 024301

(55) R. Capozza and M. Urbakh, Static friction and the dynamics of interfacial rupture, Phys. Rev. B 86 (2012) 085430

(56) N.M. Pugno, Q. Yin, X. Shi, R. Capozza, A generalization of the Coulomb's friction law: from graphene to macroscale, Meccanica 48 (2013) 8

(57) R. Capozza, N.M. Pugno, Effect of Surface Grooves on the Static Friction of an Elastic Slider, Tribol. Lett. 58 (2015) 35

(58) S.M. Rubinstein, G. Cohen, J. Fineberg, Detachment fronts and the onset of dynamic friction, Nature 430 (2004) 1005

(59) M. J. Baum, A. E. Kovalev, J. Michels, S.N. Gorb, Anisotropic Friction of the Ventral Scales in the Snake Lampropeltis getula californiae, Tribol. Lett. 54 (2014) 139

(60) V.A. Yastrebov, Sliding without slipping under Coulomb friction: opening waves and inversion of frictional force, Tribol. Lett. 62 (2016) 1 
(61) B.N. Persson, Theory of rubber friction and contact mechanics, J. Chem. Phys. 115 (2001) 8

(62) B.N. Persson, O. Albohr, U. Tartaglino, A.I. Volokitin and E. Tosatti, On the nature of surface roughness with application to contact mechanics, sealing, rubber friction and adhesion, J. Phys.: Condens. Matter 17 (2005) R1-R62

(63) J. Clarke, M. Carr, L. Damm, S. Dixon, Understanding the influence of surface roughness on the tribological interactions at the shoesurface interface in tennis, Journal of Engineering Tribology 226 (2012) 7

(64) B. Yurdumakan, N.R. Raravikar, P.M. Ajayan, A. Dhinojwala, Synthetic gecko foothairs from multiwalled carbon nanotubes, Chem. Commun. 30 (2005) 3799

(65) D. Santos, M. Spenko, A. Parness, S. Kim, M. Cutkosky, Directional adhesion for climbing: theoretical and practical considerations, Journal of Adhesion Science and Technology, 21 (2007) 1317

(66) B. Murarash, Y. Itovicha, M. Varenberg, Tuning elastomer friction by hexagonal surface patterning Soft Matters 2011,7, 5553-5557

(67) M.J. Baum, L. Heepe, E. Fadeeva, S.N. Gorb, Dry friction of microstructured polymer surfaces inspired by snake skin, Beilstein J. Nanotechnol. 5 (2014) 1091 